\title{
Paleogenetic study of ancient DNA suggestive of $X$-linked acrogigantism
}

\section{Dear Editor,}

Pituitary gigantism is caused by chronic growth hormone (GH) hypersecretion by a pituitary lesion before epiphyseal fusion. Genetic causes have been identified in nearly $50 \%$ of patients with pituitary gigantism, with germline mutations in the AIP gene being the most frequent cause (Rostomyan et al. 2015). Recently, a new form of pituitary gigantism, X-linked acrogigantism (X-LAG), was described (Trivellin et al. 2014). X-LAG is due to chromosome Xq26.3 duplication, and GPR101 is the disease-associated gene (Trivellin et al. 2014, Iacovazzo et al. 2016). X-LAG is characterized by mixed $\mathrm{GH} /$ prolactin-secreting pituitary macroadenomas and/or hyperplasia in early childhood (Beckers et al. 2015). X-LAG typically occurs sporadically in females, but somatic mosaicism also occurs in males; familial mother-to-son transmission of the Xq26.3 duplication has been reported in three familial isolated pituitary adenoma families (Trivellin et al. 2014, Daly et al. 2016, Gordon et al. 2016, Iacovazzo et al. 2016). The clinical presentation of X-LAG syndrome differs from other genetic forms of pituitary gigantism (Rostomyan et al. 2015), and many well-known historical cases of gigantism share the clinical characteristics of X-LAG syndrome (Beckers et al. 2015, Rostomyan et al. 2015). If untreated during childhood, X-LAG leads to established extreme gigantism $(>1.9 \mathrm{~m})$ before puberty (Daly et al. 2016).

We studied a historical case of severe acrogigantism. The subject, J.K., was born in 1872 in Reutlingen, in what is now Baden-Württemberg, Germany. His parents and brother were of normal size. It was reported by his doctor that J.K. had always been 'very large' and he was reputed to have a huge appetite; he measured $1.94 \mathrm{~m}$ at the age of 14 and never stopped growing thereafter (Launois \& Roy 1904). In contemporary Württemberg, the average adult male height was only $164 \mathrm{~cm}$. By the 1890s he was exhibiting himself as Giant Constantine/Le Geant Constantin (Fig. 1A). In 1898, he was $259 \mathrm{~cm}$ in height ( 8 feet 6 inches) and weighted $168 \mathrm{~kg}$ (370 pounds). He fell ill while in the Walloon region of Belgium and was hospitalized on November 15, 1901, at the Hôpital Civil in Mons, Belgium with a fever $\left(39.3^{\circ} \mathrm{C}\right)$ due to severe lower limb gangrene. Hospital records show his height as $256 \mathrm{~cm}$ and weight as $180 \mathrm{~kg}$. He improved initially after an amputation of the right leg, but the following year, he fell and the other leg was amputated below the knee. He developed post-operative septicemia and died on March 30,1902 . At autopsy, the pituitary was grossly enlarged to the size of 'a large walnut' (Launois \& Roy 1904). The sella turcica was also greatly enlarged, so much so that it was remarked that 'after removing the cerebral hemispheres and the cerebellum, the sella was so broad and deep that it brought to mind two juxtaposed spinal canals' (Launois \& Roy 1904) (Fig. 1B and $\mathrm{C}$ ). The long bones and extremities were elongated, and the proximal humeral epiphyses remained unfused (Launois \& Roy 1904). Concomitant hypogonadism (testicular atrophy) was present on examination and post-mortem. Current forensic analysis of the skeleton demonstrates bleaching of the bones consistent with reported preservation by prolonged boiling.

Given the clinical history of early-onset acrogigantism, an underlying genetic cause was thought to be likely. DNA extraction from teeth was unreliable as the skull was edentulous when originally photographed in 1904 (Launois \& Roy 1904); subsequently, teeth were added to the skull but they could not be confirmed as having been obtained from the subject himself. The skeleton was fragile after preservation by prolonged boiling, and DNA extractions from a metatarsal and the femur were unsuccessful. Based on results obtained from ancient skeletal remains, tissue from the cochlea was obtained via the petrous temporal bone (Pinhasi et al. 2015) as reported in Supplemental materials (see section on supplementary data given at the end of this article). His history of earlyonset, severe pituitary gigantism led us to suspect X-LAG (Trivellin et al. 2014, Daly et al. 2016).

The DNA sample was assayed using a ddPCR technique as previously described (Daly et al. 2016). Briefly, the ddPCR compared copy number variations $(\mathrm{CNV})$ at the GPR101 gene as compared with ZIC3, a gene that is not duplicated in X-LAG syndrome. Daly and coworkers recently showed this method was 

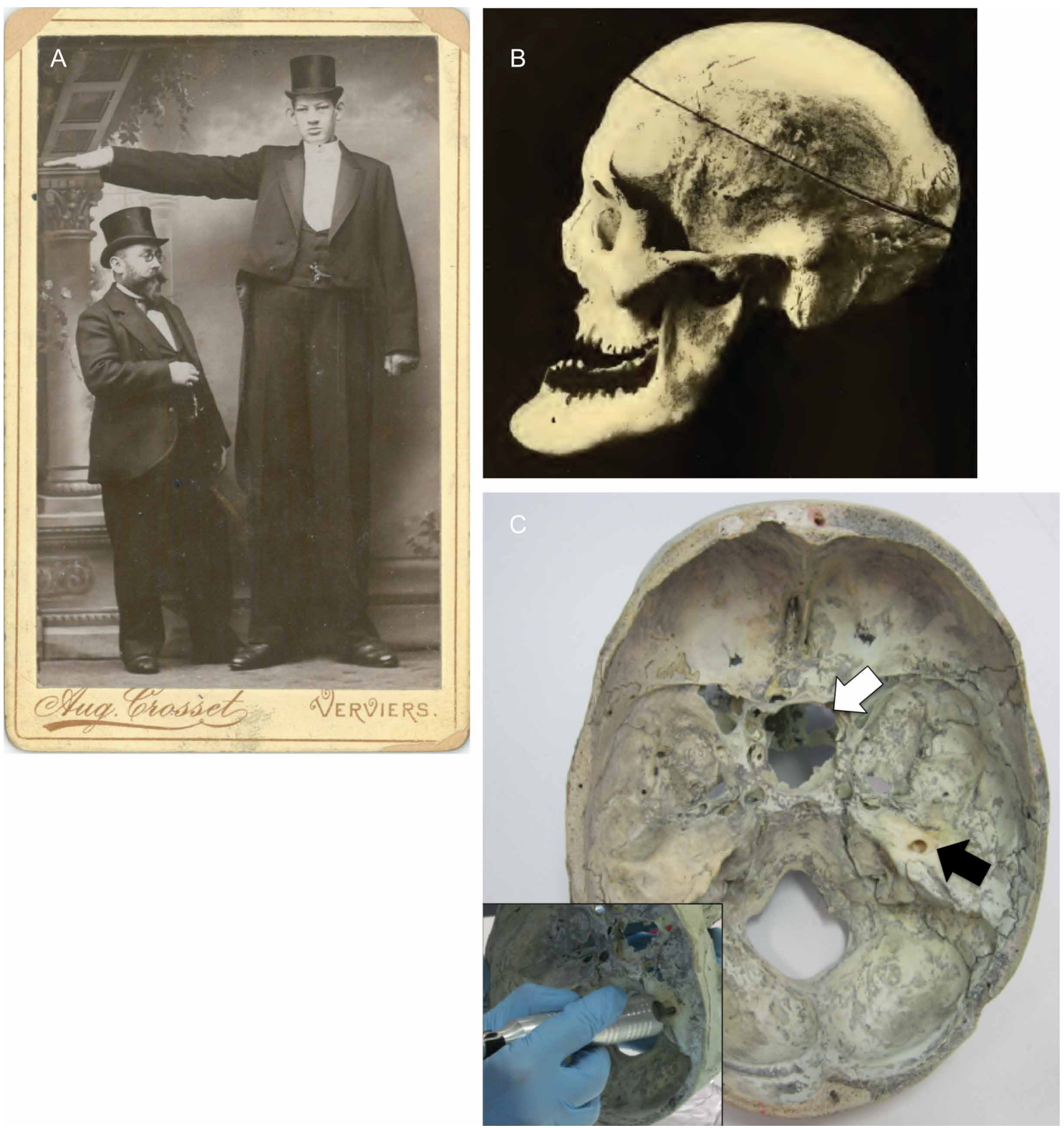

\section{Figure 1}

Panel A shows a photograph of the subject J.K. printed for publicity during his visit to Belgium immediately before his hospitalization in November 1901 at which time he was approximately $259 \mathrm{~cm}$ in height. Gigantism and acral enlargement are evident. Panel B shows the subject's skull in a lateral photograph from 1904 and illustrates marked prognathism and enlargement of the malar bones. Panel C shows an interior view of the subject's cranium and the markedly enlarged pituitary fossa/sella turcica (open arrow). DNA was obtained by drilling a small core in the petrous part of the temporal bone to reach the cochlea (inset); the process is minor and non-destructive (filled arrow).

reliable for both confirming the results of known Xq26.3 duplication carriers and non-carriers, while also identifying the duplication of GPR101 during screening of previously undiagnosed cases of X-LAG syndrome; the false-positive rate in the acrogigantism population was low (1/64 cases was borderline above the CNV minimal and maximal thresholds for duplication) (Daly et al. 2016). The study was approved by the Natural History Museum of Mons, and genetic studies regarding causes of gigantism and endocrine tumors were conducted under approval of the Ethics Committee of the Centre Hospitalier Universitaire de Liège, Belgium.

We extracted DNA from cochlear core powder, and then sequenced and post-processed it on a custom bioinformatics platform for ancient DNA. The DNA had deamination patterns consistent with that of an approximately 100-yearold sample. No pathological variants in AIP and other gigantism-associated genes were noted on DNA read

Published by Bioscientifica Ltd 
sequences. DNA was insufficient for array comparative genomic hybridization studies. The subject's DNA exceeded the statistical thresholds for GPR101 duplication (copy number variation $(\mathrm{CNV})$ value: 3.49 vs $>2.0$; Poisson $\mathrm{CNV}$ minimum value: 3.28 vs $>2.0$ ), indicating X-LAG as a likely cause of his severe pituitary acrogigantism.

The increased copy number for GPR101 strongly suggests that J.K. suffered from X-LAG syndrome. The clinical and tumoral characteristics of X-LAG are supportive of this proposed diagnosis. To achieve a height of $194 \mathrm{~cm}$ at the age of 14 years in the 1870 s required a significant period of uninterrupted overgrowth. Apart from X-LAG, few other conditions are associated with early childhood-onset pituitary gigantism. The skeleton had no evidence of McCune-Albright syndrome, and AIP mutation-associated gigantism typically begins in midadolescence (Rostomyan et al. 2015). X-LAG syndrome is associated typically with mixed GH and prolactinsecreting pituitary adenomas (with variable hyperplasia); tumors are usually macroadenomas and can be large and invasive as in the subject's case (Trivellin et al. 2014, Beckers et al. 2015). Testicular atrophy was also present in the current case that indicates significant effects of the pituitary tumor on gonadal function. This would have kept the epiphyses open, thereby contributing to the final height of $259 \mathrm{~cm}$. His proximal humeral epiphyses never fused, and his femoral epiphyses only fused late in his life, according to the autopsy accounts (Launois \& Roy 1904). Hypogonadism could also have been compounded by hyperprolactinemia, which is common in X-LAG syndrome (Trivellin et al. 2014, Beckers et al. 2015). Few adult cases of X-LAG have been characterized, so the prevalence of hypogonadism due to tumor impingement is not known, but it is a logical effect of a large tumor mass.

Advances in sequencing technologies, DNA extraction methods and bioinformatic analysis have allowed researchers to overcome challenges such as DNA authentication and contamination from modern and ancient sources and have provided access to genetic material from samples thousands of years old and originating in tropical and desert environments (Pinhasi et al. 2015, Skoglund et al. 2016). These extreme conditions are not optimal to the preservation of DNA. DNA has been retrieved from skeletal remains of other giants using techniques that have relied on DNA recovery from molar teeth. In a recent historic gigantism case, no AIP mutation was found; X-LAG was not studied but ddPCR specific to GPR101 and ZIC3 similar to that reported here could be informative (Radian et al. 2016).
As no DNA could be derived from teeth or other bony sites, we utilized the novel approach of retrieving cochlear DNA from within the petrous temporal bone as developed and validated recently in population studies (Pinhasi et al. 2015, Skoglund et al. 2016). Even in skeletal samples that are preserved by suboptimal means during preservation, retrieval of DNA and strongly supportive information that is consistent with the patient's clinical history can be obtained. This approach could be applied more generally to other possible genetic disorders that are evident on ancient or preserved skeletal remains. Also, studies similar to this case could address the utility of other approaches to $\mathrm{CNV}$ analysis such as quantitative PCR on non-amplified genomic material. The increasing number of ancient genomes that have been retrieved from phenotypically normal skeletons could provide a useful database of normal ancient DNA features and highlight the interference by environmental factors and age on the analysis and assessment of potential disease-related variants.

Valuable medical information can be gained from the genetic study of skeletal remains. Although J.K. died before the era of pituitary tumor treatment, pituitary gigantism remains a difficult-to-treat condition even today (Beckers et al. 2015, Rostomyan et al. 2015). Better understanding of the natural disease history and potential genetic causes of historical giants should reinforce the need for early effective disease control.

Albert Beckers ${ }^{1}$

Daniel Fernandes ${ }^{2,3}$ Frederic Fina ${ }^{4}$ Mario Novak ${ }^{2}$ Angelo Abati ${ }^{5}$ Liliya Rostomyan ${ }^{1}$ Albert Thiry ${ }^{6}$ L'Housine Ouafik ${ }^{4}$ Bertrand Pasture ${ }^{7}$ Ron Pinhasi ${ }^{2}$ Adrian F Daly 1

${ }^{1}$ Department of Endocrinology, Centre Hospitalier Universitaire de Liège, University of Liège, Domaine Universitaire du Sart-Tilman, Liège, Belgium

${ }^{2}$ School of Archaeology and Earth Institute, University College Dublin, Belfield, Dublin, Ireland ${ }^{3}$ Department of Life Sciences, Centro de Investigação em Antropologia e Saúde, University of Coimbra, Coimbra, Portugal 
${ }^{4}$ Assistance Publique Hôpitaux de Marseille (AP-HM) Hôpital Nord, Service de Transfert d'Oncologie Biologique, and Laboratoire de Biologie Médicale, and Aix-Marseille Université, Inserm, CRO2 UMR_S 911, Marseille, France

${ }^{5}$ Department of Legal Medicine, Centre Hospitalier

Universitaire de Liège, University of Liège, Domaine Universitaire du Sart-Tilman, Liège, Belgium ${ }^{6}$ Department of Pathology, Centre Hospitalier Universitaire de Liège, University of Liège, Domaine Universitaire du Sart-Tilman, Liège, Belgium ${ }^{7}$ Office of the Conservator, Muséum régionale des Sciences naturelles, Mons, Belgium

(Correspondence should be addressed to A Beckers; email: albert.beckers@chu.ulg.ac.be)

\section{Supplementary data}

This is linked to the online version of the paper at http://dx.doi.org/10.1530/ ERC-16-0558.

\section{Declaration of interest}

The authors declare that there is no conflict of interest that could be perceived as prejudicing the impartiality of the research reported.

\section{Funding}

The study was funded in part by grants (to A B) from the Fonds d'Investissement Pour la Recherche Scientifique (FIRS) 2014-2015, Centre Hospitalier Universitaire de Liège, Belgium and the JABBS Foundation, U.K. D F was supported by an Irish Research Council grant GOIPG/2013/36. R P was supported by a European Research Council (ERC) starting grant ADNABIOARC (263441).

\section{References}

Beckers A, Lodish MB, Trivellin G, Rostomyan L, Lee M, Faucz FR, Yuan B, Choong CS, Caberg J-H, Verrua E, et al. 2015 X-linked acrogigantism syndrome: clinical profile and therapeutic responses. Endocrine-Related Cancer 22 353-367. (doi:10.1530/ERC-15-0038)

Daly AF, Yuan B, Fina F, Caberg JH, Trivellin G, Rostomyan L, De Herder WW, Naves LA, Metzger D, Cuny T, et al. 2016 Somatic mosaicism underlies X-linked acrogigantism syndrome in sporadic male subjects. Endocrine-Related Cancer 23 221-233. (doi:10.1530/ERC-160082)

Gordon RJ, Bell J, Chung WK, David R, Oberfield SE \& Wardlaw SL 2016 Childhood acromegaly due to X-linked acrogigantism: long term follow-up. Pituitary 19 560-564. (doi:10.1007/s11102-016-0743-0)

Iacovazzo D, Caswell R, Bunce B, Jose S, Yuan B, Hernández-Ramírez LC, Kapur S, Caimari F, Evanson J, Ferraù F, et al. 2016 Germline or somatic GPR101 duplication leads to X-linked acrogigantism: a clinico-pathological and genetic study. Acta Neuropathologica Communications 4 56. (doi:10.1186/s40478-016-0328-1)

Launois PÉ \& Roy P 1904 Études Biologiques Sur Les Géants. Paris, France: Masson et Cie.

Pinhasi R, Fernandes D, Sirak K, Novak M, Connell S, AlpaslanRoodenberg S, Gerritsen F, Moiseyev V, Gromov A, Raczky P, et al. 2015 Optimal ancient DNA yields from the inner ear part of the human petrous bone. PLOS ONE 10 e0129102. (doi:10.1371/journal. pone.0129102)

Radian S, Diekmann Y, Gabrovska P, Holland B, Bradley L, Wallace H, Stals K, Bussell A-M, McGurren K, Cuesta M, et al. 2016 Increased population risk of AIP-related acromegaly and gigantism in Ireland. Human Mutation 38 78-85. (doi:10.1002/humu.23121)

Rostomyan L, Daly AF, Petrossians P, Nachev E, Lila AR, Lecoq A-L, Lecumberri B, Trivellin G, Salvatori R, Moraitis AG, et al. 2015 Clinical and genetic characterization of pituitary gigantism: An international collaborative study in 208 patients. Endocrine-Related Cancer 22 745-757. (doi:10.1530/ERC-15-0320)

Skoglund P, Posth C, Sirak K, Spriggs M, Valentin F, Bedford S, Clark GR, Reepmeyer C, Petchey F, Fernandes D, et al. 2016 Genomic insights into the peopling of the Southwest Pacific. Nature 538 510-513. (doi:10.1038/nature19844)

Trivellin G, Daly AF, Faucz FR, Yuan B, Rostomyan L, Larco DO, Schernthaner-Reiter MH, Szarek E, Leal LF, Caberg J-H, et al. 2014 Gigantism and acromegaly due to Xq26 microduplications and GPR101 mutation. New England Journal of Medicine 371 2363-2374. (doi:10.1056/NEJMoa1408028)

Received in final form 22 December 2016

Accepted 3 January 2017

Accepted Preprint published online 3 January 2017
(C) 2017 Society for Endocrinology Printed in Great Britain
Published by Bioscientifica Ltd 